\title{
Calcified Fibroma of the Ovary.
}

\author{
By G. A. Casalis, M.B., etc., \\ Gynacological Surgeon to the Victoria Hospital, Wynberg, \\ Cape Town.
}

ThE rarity of calcified fibrous growths of the ovaries gives some interest to the following observation :-Mary N., aged 42, multipara, consulted me on November 12, 1907, for profuse memstrual and intermenstrual hæmorrhages accompanied by severe pains in the lumbar and hypo-gastric regions. The pains had of late become continuous, and the patient was seldom free for more than a week of the menstrual discharge. On examination the patient, a fat coloured woman, was found to suffer from a fibroma of the uterus, with what appeared, to the examining fingers, to be a second and pedunculated tumour arising from the fundus.

A laparatomy revealed the presence of an ordinary interstitial fibroma which had invaded the whole of the uterine body and was about the size of a cocoa-nut. The pedunculated growth proved to be a calcified ovarian fibroma as big as a cricket ball and quite distinct from the uterine tumour, to which it had preserved its usual relation. The ovarian growth was white and as hard as a stone-a median section performed with a fine saw showing the fibroma to be fully and totally infiltrated by the lime salts-indeed, except for a peculiarly tough fibrous covering, very little fibrous tissue was left, especially in the centre which was uniformally stony and calcareous. The Fallopian tube stretching over the growth was hypertrophied, but otherwise normal, the fimbrix being fleshy and well developed. The canal was patent up to the uterine insertion, when it became lost in the uterine growth. The latter weighed about 2 lbs., the ovarian growth $1 \mathrm{lb} .4$ ounces. The right ovary was micro-cystic, the tube healthy, and both were preserved when the uterine and left ovarian tumours were excised. 